\title{
MEJORAMIENTO PARTICIPATIVO EN MAÍZ: SU CONTRIBUCIÓN EN EL EMPODERAMIENTO COMUNITARIO EN EL MUNICIPIO DE MUQUI, BRASIL 1
}

\author{
Altair Toledo-Machado², José Arcanjo-Nunes ${ }^{3}$, Cynthia Torres de Toledo Machado ${ }^{2}$, Luciano Lourenço-Nass ${ }^{4}$, \\ Franknély Candido-da Rocha Bettero 5
}

\begin{abstract}
RESUMEN
Mejoramiento participativo en maíz: su contribución en el empoderamiento comunitario en el municipio de Muqui, Brasil. El desarrollo de la estrategia del mejoramiento participativo, en las comunidades de pequeños agricultores ubicados en el municipio de Muqui, en el Estado de Espírito Santo, Brasil, influyó en la autosostenibilidad de la producción de semilla y en la obtención de variedades de maíz mejor adaptadas a sus sistemas de manejo Los agricultores de esta región vienen desarrollando, desde 1993, trabajos en el campo comunitario de semillas. A partir de 2001, como estrategias del mejoramiento participativo fueron desarrollados trabajos referentes al rescate de variedades locales y mejoradas de maíz, y ensayos de evaluación de variedades. Diez variedades fueron utilizadas en este trabajo: Sol da Manhã , Eldorado, BR 106, BR 473, BR 451, Saracura, Caiano de Sobrália, Palha Rôxa de Santa Catarina, Caiano de Espírito Santo y Fortaleza. Con excepción de las variedades BR 106, BR 473, BR 451 y Saracura, que fueron seleccionadas de forma convencional, las demás fueron seleccionadas con base en fitomejoramiento participativo. Fueron realizados tres ciclos de selección en cada una de esas variedades y las mismas fueron evaluadas en los años agrícolas de 2002/2003, 2003/2004 y 2004/2005, en ensayos en bloques al azar, con tres repeticiones y manejo orgánico. Las actividades de mejoramiento, en las comunidades de Muqui, fueran realizadas enfatizando la variedad Fortaleza, se empleó el esquema de selección masal estratificada. Los campos de semillas comunitarias se incrementaron, tanto en productividad como en la mayor participación de los agricultores, además de la participación en los ensayos y los campos de mejoramiento.
\end{abstract}

Palabras clave: Agrobiodiversidad, agroecología, desarrollo sostenible, variedades locales, Zea mays.

\begin{abstract}
Participatory breeding contribution for small farmer empowerment in Muqui Municipality, Brazil. The strategy of participatory breeding was highly successful for the empowerment of the small farmer communities in Muqui, State of Espírito Santo, Brazil. Since 1993, local farmers have been developing experiences with communityowned seed fields. From 2001, this work was improved by using participatory methodologies for seed production. The strategy of participatory breeding includes the rescue of local and improved varieties, and evaluation trials of different varieties. Ten varieties were used in this study: 'Sol da Manhã ', 'Eldorado', 'BR 106', 'BR 473', 'BR 451', 'Saracura', 'Caiano de Sobrália', 'Palha Rôxa de Santa Catarina', 'Caiano de Espírito Santo', and 'Fortaleza'. These varieties were selected using participatory approaches by different communities in Brazil, except 'BR 106', 'BR 473', 'BR 451', and 'Saracura' that were obtained by conventional breeding. Three cycles of stratified mass selection were used for each variety. The varieties were evaluated using complete randomized block designs, with three replications, under organic management during the 2002/2003, $2003 / 2004$, and 2004/2005 growing seasons. Breeding activities developed by the local communities of Muqui concentrated their efforts on 'Fortaleza' variety (the variety' names derived from a local community). The yield in communal seed fields was increased by two factors: increase in productivity and higher farmer's participation.
\end{abstract}

Key words: Agrobiodiversity, agroecology, sustainable development, local varieties, Zea mays.

\footnotetext{
1 Recibido: 7 de noviembre, 2005. Aceptado: 5 de junio, 2006.

2 Embrapa Cerrados. Correo electrónico: altair@cpac.embrapa.br; cynthia@cpac.embrapa.br

3 Municipalidad de Muqui, UFES. Correo electrónico: nunesarcanjo@terra.com.br

4 Embrapa Recursos Genéticos y Biotecnología. Correo electrónico: luciano.nass@ars.usda.gov

5 Técnico en Ciencias Agropecuarias y miembro de la Comunidad Fortaleza en Muqui.
} 


\section{INTRODUCCIÓN}

El empoderamiento (o autoindependencia en las gestiones de desarrollo comunitario) de las comunidades donde habitan pequeños agricultores puede hacerse a partir del manejo sostenible de la agrobiodiversidad dentro de sistemas agroecológicos. Si se considera que la pérdida de sistemas de cultivo y de variedades locales hacen que los valores (p. ej. culturales y sociales) sean igualmente perdidos.

Como consecuencia de los procesos de erosión se verifica el empobrecimiento de las comunidades de agricultores de subsistencia. En este sentido, el manejo de los recursos vegetales, incluyendo el mejoramiento vegetal participativo, desempeña un papel relevante en esas comunidades, donde además de las pérdidas descritas, son comunes las condiciones adversas debido a problemas ambientales, climáticos y económicos.

La estrategia del mejoramiento participativo con enfoque en la agrobiodiversidad puede contribuir a la construcción de un ambiente agrícola sostenible, con la elevación de renta y agregación de valores ambientales y sociales, estableciendo las bases para la soberanía alimentaria de las comunidades, que pasan a tener autonomía en la producción de las semillas (Machado 1998; Machado 2000; Almekinders y Elings 2001; Machado y Fernández, 2001; Sperling et al. 2001; Witcombe et al. 2001; Smith et al. 2001; Bänziger y Cooper, 2001; Machado et al. 2002; Machado y Machado, 2003; 2004).

Dentro de las experiencias con trabajos de mejoramiento participativo se puede citar la desarrollada con maíz en 1990, cuyo trabajo conjunto con enfoque participativo envolucró instituciones públicas, representadas por la Empresa Brasileira de Pesquisa Agropecuária (EMBRAPA), la Red PTA (red de organizaciones nogubernamentales, que trabajan con pequeños productores de diferentes partes del Brasil) y a pequeños agricultores de la comunidad "Sol da Manhã " en el Estado de Río de Janeiro. La primera acción de ese trabajo fue el rescate y caracterización de variedades locales de maíz (Zea mays L.), debido al que muchas de esas variedades estaban en extinción y siendo sustituidas por variedades mejoradas e híbridos. La segunda acción fue la evaluación de esas variedades en ensayos de rendimiento y la tercera fue el mejoramiento de esas variedades, con la participación de agricultores dentro de un enfoque integrado (Machado y Machado 2004).
Como consecuencia de ese trabajo en Red iniciado en 1990, algunos trabajos de mejoramiento participativo en maíz en el Brasil, se incrementaron y fortalecieron, como los desarrollados por comunidades en el oeste de Santa Catarina, en los cuales más de cien variedades de maíz se rescataron y por lo menos quince están en proceso de mejoramiento y producción comunitaria (Canci 2002 y Canci et al. 2004). Un proceso muy parecido se desarrolló en el Paraná y fue coordinado por la AS-PTA. En Espírito Santo, en Muqui, dicho trabajo de mejoramiento participativo recibió apoyo de la municipalidad y organizaciones locales (Machado et al. 2002).

Otras experiencias de mejoramiento participativo se concretaron en el norte de Minas y en el centro oeste del Brasil. En todas ellas se constató que el rescate de variedades locales de maíz fue una estrategia necesaria, no solamente para preservar su diversidad, sino también para estudiar su estructura, verificando el proceso de erosión genética que puede haber ocurrido y su potencial para uso inmediato o futuro.

Se torna fundamental que las variedades, después de rescatadas, sean sometidas a experimentación en diferentes localidades y por varios años, para determinar su valor como variedad local, permitiendo la difusión de las mismas a través del cambio de materiales entre los agricultores, o evaluar su potencial genético para mejoramiento, validando su uso en diferentes agro-ecosistemas. Así, la evaluación de diferentes variedades locales de maíz en diferentes agro-ecosistemas puede aportar importantes fuentes genéticas para varios tipos de estrés ambiental bióticos (plagas, enfermedades, malezas) o abióticos.

El desarrollo de estrategias de mejoramiento participativo en sistemas agro-ecológicos se torna fundamental principalmente cuando se busca la sostenibilidad y el empoderamiento de comunidades locales (Machado y Machado 2003). El presente estudio tiene como objetivo conocer la dinámica de diferentes variedades de maíz, cuando se someten a diferentes formas de mejoramiento incluyendo el sistema de mejoramiento participativo con visión agro-ecológica, identificando las relaciones genético-ecológicas que se establecen y sus procesos, para que las comunidades puedan tener un incremento en la producción de forma sostenible con un mínimo impacto ambiental y social y con un uso mínimo de insumos externos, buscando su autonomía y fortaleciendo su base social. 


\section{MATERIALES Y MÉTODOS}

Las actividades de mejoramiento participativo se realizaron en la Comunidad Fortaleza localizada en Muqui, en el estado de Espírito Santo (ES), en la región sudeste del Brasil, a partir de una estrategia de polos irradiadores. Todas las actividades referentes al manejo de la agro-biodiversidad en la cual se incluye el rescate, evaluación, mejoramiento participativo y producción de semillas comunitaria, se realizaron en la Comunidad Fortaleza (polo), contando con la presencia de representantes de otras comunidades de Muqui (Santa Rita, Demanda, San Luis y Siempre Viva) y del asentamiento de Reforma Agraria "17 de Abril".

En 1992, diferentes comunidades de Espírito Santo con actividades participativas se involucraron con la Red Nacional de Semillas (Soares et al. 1998). En la Comunidad Fortaleza, el trabajo se inició en 1993 a partir de campos comunitarios de semillas, y, a partir de 2001, se fortaleció por la Municipalidad de Muqui junto con la EMBRAPA. Los agricultores de esta comunidad poseen una amplia experiencia en investigación participativa y transmiten estas experiencias a otras comunidades, del estado y para el Brasil. Además de ésto, promueven actividades urbanas dentro de la ciudad de Muqui en forma de ferias orgánicas, actividades agroindustriales, produciendo harinas de maíz, polentas (pasta de harina de maíz con agua) congelada y otros productos. Los agricultores cuentan con el apoyo de la Municipalidad, que otorga el sello de calidad, realizan estas actividades en las escuelas para los niños y jóvenes, que luego las incorporan en fiestas y desfiles escolares.

\section{Estrategia de trabajo}

La estrategia adoptada en este trabajo fue el manejo de la agro-biodiversidad con enfoque agro-ecológico (Machado y Machado 2003) en el cual se incluyeron las actividades de rescate de germoplasma vegetal, ensayos de evaluación, mejoramiento participativo, producción de semillas y conservación, siempre en ambientes con manejo agro-ecológico. Tales actividades se describen a continuación:

\section{Rescate}

El rescate se realizó con la finalidad de incrementar la diversidad genética de variedades de maíz en la región de Muqui, tratando de encontrar variedades adaptadas a este agro-ecosistema. Dentro de esta estrategia el rescate se direccionó en busca de variedades locales de otras regiones brasileñas sometidas a procesos de mejoramiento participativo y variedades mejoradas de centros de investigación.

Las siguientes variedades fueron rescatadas: Palha Rôxa de Santa Catarina, variedad seleccionada por la Comunidad de Anchieta, en el oeste del estado de Santa Catarina (SC); Caiano de Sobrália, variedad seleccionada por la comunidad de Sobrália, en el estado de Minas Gerais (MG); Caiano de Espírito Santo, variedad seleccionada por comunidades de Alegre, en el estado do Espírito Santo (ES); Sol da Manhã y Eldorado, variedades con un largo proceso de mejoramiento participativo, involucrando la Comunidad Sol da Manhã , de Seropédica, en el estado de Río de Janeiro (RJ), y la EMBRAPA, donde la variedad Eldorado se ha seleccionado para el sistema orgánico de producción; BR 106, BR 473, BR 451 y Saracura son variedades desarrolladas por la EMBRAPA. Estas variedades fueron seleccionadas de forma convencional y, finalmente, la variedad Fortaleza se viene seleccionando desde hace 12 años por Claudio Evanes Bettero Candido, de la Comunidad Fortaleza. Este rescate se realizó para obtener muestras de $2 \mathrm{~kg}$ representadas por 200 espigas, siendo retiradas 100 semillas por espiga.

\section{Ensayos de evaluación}

Los objetivos de los ensayos de evaluación fueron: conocer la variabilidad genética de las variedades rescatadas; verificar su adaptación al medio ambiente local y al sistema de producción agro-ecológica; cambio de experiencias entre agricultores de diferentes comunidades, oficina de aprendizaje para agricultores, técnicos, estudiantes y otros sobre la diversidad genética de maíz; encuentro festivo de la comunidad. Las variedades que formaron parte de los ensayos de rendimiento fueron: 
1. Sol da Manhã : población de granos duros y semi-duros, anaranjados, con segregación para blanco y predominio de germoplasma Cateto, Eto y Duros del Caribe, originada de 36 poblaciones de América Central y América del Sur (Machado et al. 1992).

2. Eldorado: población de granos dentados y semi-dentados, amarillos con segregación para blanco y predominio de la raza Tuxpeño, formada a partir de poblaciones de México, América Central y América del Sur (Machado et al. 1992).

3. BR 106: variedad obtenida en la Embrapa maíz y sorgo, en Siete Lagunas, MG, a partir de tres cultivares brasileños (Maya, Centralmex y Dentado Compuesto) y una introducción exótica (Tuxpeño 1). Presenta endosperma dentado y coloración amarilla, porte bajo (carácter poligénico) (Naspolini et al. 1981).

4. BR 473: sintético de ciclo precoz, de granos amarillos, semiduros, con alta calidad proteica, generada por el inter-cruzamiento de seis líneas élite QPM. En los últimos dos ciclos de selección de progenies de medios-hermanos, se seleccionó para aumentar la adaptación amplia, a las condiciones del Sudeste y Sur del Brasil (Machado et al. 2002).

5. BR 451: sintético de ciclo precoz, de granos blancos, dentados, con alta calidad proteica. Población introducida del CIMMYT, en 1983, como Blanco Dentado (Machado et al. 1998a).

6. Saracura: población de ciclo semi-precoz, de granos anaranjados, semi-duros, con 13 ciclos de selección recurrente para tolerancia al encharcamiento del suelo.

7. Caiano de Sobrália: variedad de granos dentados, amarillos de ciclo semi-precoz y con varios ciclos de selección masal estratificada realizada por la comunidad de Sobrália (MG) (Machado 1998).

8. Palha Rôxa de Santa Catarina: variedad de granos dentados, porte alto, coloración amarillo arrochiado. Variedad plantada en Anchieta (SC) hace más de 20 años (Canci et al. 2004).

9. Caiano de Espírito Santo: variedad de granos dentados, amarillos de ciclo tardío. Variedad sembrada en Alegre (ES) hace más de 20 años (Machado 1998).
10. Fortaleza: Variedad originaria de la BR 106, posee germoplasma y se cultiva en la región de Muqui desde hace más de 15 años, con varios ciclos de selección masal estratificada.

Para la realización de ese trabajo fueron considerados tres ciclos de selección para cada una de las variedades antes citadas, así denominados: $\mathrm{C}_{0}-$ Ciclo Cero - que corresponde a la variedad obtenida en el año de 2002, $C_{1}$ - Ciclo Uno - corresponde a un ciclo de selección masal estratificada seleccionada en 2002 y obtenida en 2003 y $\mathrm{C}_{2}$ - Ciclo Dos - corresponde al segundo ciclo de selección masal estratificada seleccionadas en 2003 y obtenida en 2004.

Las evaluaciones de esas variedades se hicieron en tres años agrícolas diferentes: 2002/2003; 2003/2004 y 2004/2005. El diseño experimental utilizado fue el de bloques al azar con tres repeticiones. Los experimentos se condujeron en una misma área en la Comunidad Fortaleza en Muqui, ES. La parcela experimental estuvo constituida por dos hileras de $5 \mathrm{~m}$ con $1 \mathrm{~m}$ de distancia entre hileras. Se sembraron 76 semillas, para que después del raleo o aclareo cada parcela tuviese 52 plantas. Se utilizó un bordo compuesto de la mezcla de las poblaciones, sembrando siete semillas/metro, sin aclareo.

El área donde se condujeron los experimentos se manejó como sistema orgánico, donde se aplicaron 10 toneladas de estiércol por hectárea y abonos verdes. Este manejo comenzó después de la cosecha del ensayo realizado en el primer año agrícola. El análisis de suelos para los tres años agrícolas se encuentra en el Cuadro 1.

Se evaluaron las siguientes características agronómicas: (1) floración masculina (días); (2) floración femenina (días); (3) altura de la planta (metros); (4) altura de la espiga (metros); (5) plantas quebradas y acamadas (registradas en porcentaje en la parcela); (6) número de plantas (para la corrección del stand); (7) número total de espigas de la parcela; (8) peso de espigas (kg/parcela) y (9) humedad de los granos (\%) (media de la parcela). La humedad del grano se corrigió al $15 \%$.

Se adoptó, en este estudio, un modelo fijo, en el cual los tratamientos no son considerados como una muestra al azar de una población. Todos los ensayos de variedades o poblaciones de maíz constituyeron un conjunto fijo, porque las variedades no representan una 
Cuadro 1. Análisis de suelo para los experimentos conducidos en la Comunidad Fortaleza, Brasil en los años agrícolas de 2002/2003, 2003/2004 y 2004/2005.

\begin{tabular}{lccc}
\hline \multirow{2}{*}{ Características químicas } & \multicolumn{3}{c}{ Años agrícolas } \\
\cline { 2 - 4 } & $\mathbf{2 0 0 2 / 2 0 0 3}$ & $\mathbf{2 0 0 3} / \mathbf{2 0 0 4}$ & $\mathbf{2 0 0 4} / \mathbf{2 0 0 5}$ \\
\hline pH en agua & 5,8 & 5,9 & 6,9 \\
Al $\left(\mathrm{cmolc} \cdot \mathrm{dm}^{-3}\right)$ & 0,0 & 0,0 & 0,0 \\
$\mathrm{H}+\mathrm{Al}\left(\mathrm{cmolc}^{\prime} \cdot \mathrm{dm}^{-3}\right)$ & 3,9 & 1,76 & 1,30 \\
P $\left(\mathrm{mg} \cdot \mathrm{dm}^{-3}\right)$ & 17,0 & 18,24 & 24,0 \\
$\mathrm{~K}\left(\mathrm{mg} \cdot \mathrm{dm}^{-3}\right)$ & 62,0 & 140,0 & 224,0 \\
$\mathrm{Ca}\left(\mathrm{cmolc} \cdot \mathrm{dm}^{-3}\right)$ & 4,6 & 1,77 & 7,5 \\
$\mathrm{Mg}\left(\mathrm{cmolc} \cdot \mathrm{dm}^{-3}\right)$ & 1,8 & 0,85 & 1,7 \\
$\mathrm{Materia} \mathrm{orgánica}\left(\mathrm{dag} \cdot \mathrm{kg}^{-1}\right)$ & 2,69 & 1,80 & 4,30 \\
$\mathrm{~B}\left(\mathrm{mg} \cdot \mathrm{dm}^{-3}\right)$ & - & 0,53 & 0,4 \\
$\mathrm{Cu}\left(\mathrm{mg} \cdot \mathrm{dm}^{-3}\right)$ & - & 1,70 & 1,20 \\
$\mathrm{Fe}\left(\mathrm{mg} \cdot \mathrm{dm}^{-3}\right)$ & - & 174 & 100 \\
$\mathrm{Mn}\left(\mathrm{mg} \cdot \mathrm{dm}^{-3}\right)$ & - & 75 & 15 \\
$\mathrm{Zn}\left(\mathrm{mg} \cdot \mathrm{dm}^{-3}\right)$ & - & 2,6 & 2,4 \\
\hline
\end{tabular}

población de variedades. Así, las conclusiones derivadas de ese experimento son válidas sólo para el conjunto analizado, no siendo posible la extrapolación para otro conjunto de variedades. Los procedimientos estadísticos constarán de análisis de varianza con aplicación de una prueba de F de Snedecor. Para las fuentes de variación donde existió diferencia significativa, se aplicó la prueba DMS (Diferencia mínima significativa).

Para los caracteres floración masculina y femenina, altura de planta, altura de espiga y peso de espigas, los cuadrados medios residuales no variaron mucho (hasta cuatro veces entre el mayor y el menor). Los análisis de varianza conjunta se hicieron de acuerdo con Gomes (1982).

\section{Mejoramiento varietal}

Las variedades sometidas al esquema de mejoramiento participativo en este trabajo fueron: Sol da Manhã, Eldorado, Palha Rôxa de Santa Catarina y Fortaleza.

La variedad Sol da Manhã fue seleccionada en la comunidad Cunha localizada en la Ciudad Ocidental, en el estado de Goiás (GO); la variedad Eldorado se seleccionó en el Sitio Alegría con apoyo de la ONG Ecoidéia y de la Asociación de Agricultores Ecológicos del Distrito Federal (DF) y entorno (esta variedad se seleccionó en sistema orgánico); la variedad Palha Rôxa de Santa Catarina fue seleccionada en la comunidad de Anchieta (SC); y la variedad Fortaleza fue seleccionada en la Comunidad Fortaleza en Muqui (ES).

En los trabajos con las variedades Sol da Manhã y Eldorado hubo la participación de la Embrapa Cerrados, para la Palha Rôxa hubo la participación de la Universidad Federal de Santa Catarina y, para la variedad Fortaleza hubo la participación de la Municipalidad de Muqui con apoyo de la EMBRAPA.

El método de selección utilizado en esas variedades fue el de selección masal estratificada propuesto por Paterniani (1990) y adaptado para cada localidad obedeciendo al siguiente delineamento de siembra y selección: se plantó un lote aislado con 3.000 plantas, el cual posteriormente se subdividió en 60 estratos (subparcelas) de $10 \mathrm{~m}$ con aproximadamente 50 plantas por estrato.

La selección se realizó dentro de cada estrato. Se utilizó una intensidad de selección del 20\% quedando, así, 10 plantas seleccionadas por estrato resultando un total de 600 plantas seleccionadas. Para la selección de plantas se dio énfasis a la selección de plantas con competencia completa, no caídas y no quebradas, de porte bajo, sanas, espigas bien empajadas, además de otras características favorables.

A partir de 600 espigas seleccionadas se escogieron las 200 mejores, las cuales sirvieron de base para la próxima siembra. Para asegurar un muestreo adecuado, se retiró el mismo número de semillas de cada espiga, rescatando de esta manera, el número original de semillas. La selección se realizó de forma integrada y participativa.

Las variedades Caiano de Sobrália y Caiano de Espírito Santo se seleccionaron por los agricultores a partir de selección masal simples y las variedades BR 106, BR 473, BR 451 y Saracura fueron seleccionadas por la Embrapa Maíz y Sorgo a partir de selección masal estratificada conforme se describió anteriormente, pero de forma convencional. 
En este trabajo se calculó la ganancia de selección (GS) de forma simples para familias seleccionadas de forma masal, conforme a Vencovsky (1987), quien propuso que $\mathrm{GS}=\mathrm{X}_{\mathrm{M}}-\mathrm{X}_{\mathrm{F}}$, donde $\mathrm{X}_{\mathrm{F}}=$ la media del carácter en el material que está en el campo, antes de la selección; $X_{M}=$ la media del carácter en la población mejorada, en la generación siguiente, después de la multiplicación.

\section{Producción de semillas comunitarias}

El trabajo de producción de semillas en Muqui siempre estuvo vinculado al trabajo de mejoramiento participativo. Actualmente las comunidades están produciendo semillas de las variedades Fortaleza y Sol da Manhã . Dentro de los cuidados adoptados por las comunidades están la elección del área y el aislamiento del campo para evitar contaminación. El almacenamiento es hecho en sacarías y tambores de 200 litros herméticamente cerrados.

\section{RESULTADOS Y DISCUSIÓN}

El desarrollo de este trabajo tuvo por objetivo encontrar estrategias para el fortalecimiento de la Comunidad Fortaleza, en el Municipio de Muqui (ES). Esta comunidad se encuentra en una región montañosa con predominio de tierras de baja fertilidad y con una agricultura típicamente familiar. Esta localidad sufrió mucho con la modernización de la agricultura y con su consecuente industrialización, hecho que generó un sistema de dependencia de insumos agrícolas. Hubo un fuerte impacto en la biodiversidad local y en el empoderamiento de los agricultores.

Esta situación de dependencia debida a la industrialización y a la pérdida de variedades locales ha sido abordada por diferentes autores (Vernooy y Song 2004). En ese sentido, el desarrollo de la investigación participativa se tornó necesaria y la estrategia inicial fue de apropiar la comunidad con experimentación, técnicas de mejoramiento, producción de semillas y manejo de los suelos, buscando así la sostenibilidad de esa comunidad y el empoderamiento de sus miembros.

Este trabajo inició con un fuerte apoyo de la municipalidad y, en un primer momento, la comunidad definió como estrategia prioritaria el desarrollo de ese trabajo en el cultivo del maíz. Para realizar la experimentación se seleccionó una área comunitaria, la cual fue monitoreada por los años agrícolas siguientes, a través del análisis de los suelos. Esta área fue manejada orgánicamente y se pudo observar la evolución de la fertilidad durante los tres años de experimentación agrícola realizada en esa localidad (Cuadro 1), cuando se verificó un incremento en la disponibilidad de fósforo $(\mathrm{P})$, potasio $(\mathrm{K})$ y de materia orgánica $(\mathrm{MO})$ y reducción en la acidez potencial del suelo $(\mathrm{H}+\mathrm{Al})$.

Se logró rescatar algunas variedades de maíz, según criterio de informaciones técnicas y agronómicas, con base en datos de ensayos de rendimiento realizados. En este sentido, las variedades Palha Rôxa de Santa Catarina, Caiano de Espírito Santo, Caiano de Sobrália fueran seleccionadas en función de los datos obtenidos de ensayos realizados en diferentes regiones del Brasil y relatados en Soares et al. (1998). Las variedades Sol da Manhã y Eldorado son variedades generadas mediante mejoramiento participativo, así como la variedad Fortaleza.

Las variedades BR 106, BR 473, BR 451 y Saracura son variedades comerciales. La variedad Fortaleza está siendo mejorada en la Comunidad Fortaleza desde 1993. Sin embargo, a partir de 2001 este trabajo pasó a ser sistematizado y acompañado por la investigación. Por el acompañamiento de los campos de mejoramiento y producción la comunidad viene adquiriendo conocimiento y sigue incrementando su producción.

El mejoramiento participativo es una nueva vertiente del mejoramiento genético vegetal donde uno de los ingredientes fundamentales es la inclusión sistemática de los conocimientos, habilidades, experiencias, prácticas y preferencia de los agricultores (Consultive Group on International Agricultural Research 1999a, 1999b). Se realizaron diferentes ensayos de rendimiento para un mejor entendimiento de la cultura del maíz, en relación con su diversidad y adaptabilidad.

La experimentación es una forma de motivación y de capacitación para los agricultores, si los resultados son acordes a sus necesidades (Machado et al. 2002). En la conducción de los ensayos que serán relatados, hubo participación efectiva de los productores en todas las etapas del proceso. Se implementó un foro 
constante de discusión y debates antes de la instalación y después de la cosecha de los experimentos. Se capacitó a los agricultores en aspectos técnicos y metodológicos de la experimentación.

Estos ensayos se efectuaron en los años agrícolas de 2002/2003, 2003/2004 y 2004/2005. Se efectuó un análisis conjunto para todos los caracteres, excepto para plantas acamadas o caídas, y que fueron registrados en porcentaje. En el Cuadro 2 se presentan los datos referentes al análisis conjunto, donde se observa la significancia estadística al $1 \%$ por la prueba de F para todos los caracteres en lo que se refiere a años y variedades.

Estos resultados demuestran que hubo diferencia significativa entre los ensayos realizados en los diferentes años agrícolas, bien como hubo diferencia entre las variedades para todos los caracteres. Para la interacción de años $\mathrm{x}$ variedades no fue significativa para todos los caracteres, ésto se explica porqué el comportamiento de las variedades fue semejante en los diferentes años.

En el Cuadro 3 se presentan las medias referentes a los caracteres evaluados a partir del análisis conjunto para los años agrícolas de 2002/2003, 2003/2004 y 2004/2005. Para floración masculina, las variedades BR 451 y Sol da Manhã presentaron los menores valores con 52 y 54 días respectivamente, y las variedades Palha Rôxa (61 días) y Caiano do Espírito Santo (62 días) presentaron los valores más altos. Para

Cuadro 2. Análisis conjunta para los caracteres floración masculina (FM), floración femenina (FF), altura de planta (AP), altura de espiga (AE) y peso de espigas de maíz en Muqui, Brasil, referente a los años de 2002/2003, 2003/2004 y 2004/2005.

\begin{tabular}{lrrrrrr}
\hline Fuentes de & \multicolumn{6}{c}{ Cuadrados medios $(\mathbf{Q M})$} \\
\cline { 2 - 7 } variación (FV) & $\mathbf{G L}^{*}$ & FM & FF & AP & AE & Peso \\
\hline Años (A) & 2 & $* *$ & $* *$ & $* *$ & $* *$ & $* *$ \\
Variedades (V) & 9 & $* *$ & $* *$ & $* *$ & $* *$ & $* *$ \\
A x V & 18 & $\mathrm{~ns}$ & $\mathrm{~ns}$ & $\mathrm{~ns}$ & $\mathrm{Ns}$ & $\mathrm{ns}$ \\
Residuo & 54 & & & & & \\
\hline
\end{tabular}

* GL: grado de libertad; ** Significativo al 1\% de significancia mediante la prueba de F; ns: No significativo.
Cuadro 3. Medias referentes a los caracteres de floración masculina (FM), floración femenina (FF), altura de planta (AP), altura de espiga (AE), plantas acamadas y quebradas (PT/PQ) y peso de espigas de maíz, a partir del análisis conjunto para los años agrícolas de 2002/2003; 2003/2004 e 2004/2005.

\begin{tabular}{lcccccc}
\hline \multicolumn{1}{c}{ Variedades } & FM & FF & AP & AE & PT/PQ & Peso \\
& $($ días) & $($ días $)$ & $(\mathbf{m})$ & $(\mathbf{m})$ & $(\%)$ & $(\mathbf{k g} / \mathbf{h a})$ \\
\hline Sol da Manhã & 54 & 58 & 2,42 & 1,42 & 14 & 6.984 \\
Eldorado & 57 & 60 & 2,70 & 1,63 & 15 & 9.354 \\
BR 106 & 58 & 63 & 2,71 & 1,52 & 11 & 7.773 \\
BR 473 & 56 & 60 & 2,55 & 1,42 & 19 & 6.591 \\
BR 451 & 52 & 57 & 2,35 & 1,11 & 26 & 6.192 \\
Saracura & 56 & 61 & 2,52 & 1,49 & 24 & 6.654 \\
Caiano de Sobrália & 59 & 64 & 2,60 & 1,49 & 12 & 8.488 \\
Palha Rôxa de & & & & & & \\
Santa Catarina & 61 & 66 & 3,06 & 1,93 & 18 & 7.756 \\
Caiano de Espírito & & & & & & \\
Santo & 62 & 67 & 3,03 & 2,04 & 12 & 7.188 \\
Fortaleza & 60 & 64 & 2,62 & 1,58 & 14 & 8.617 \\
& & & & & & \\
Media General & 57,5 & 62 & 2,66 & 1,56 & 16,5 & 7.560 \\
CV (\%) & 2,83 & 2,77 & 7,37 & 12,53 & & 13,67 \\
DMS (5\%) & 3,00 & 3,08 & 0,47 & 0,25 & & 1.891 \\
\hline
\end{tabular}

floración femenina, BR 451 y Sol da Manhã también presentaron los menores valores con 57 y 58 días, respectivamente, mientras que Palha Rôxa y Caiano de Espírito Santo fueron las más tardías con 66 y 67 días, respectivamente.

Para altura de planta (AP) y altura de espiga (AE) las variedades BR 451 y Sol da Manhã presentaran los menores valores de 2,35 $\mathrm{m}$ y $2,42 \mathrm{~m}$ para AP y $1,11 \mathrm{~m}$ y $1,42 \mathrm{~m}$ para $\mathrm{AE}$, respectivamente. Los mayores valores fueron presentados por las variedades Palha Rôxa y Caiano con $3,06 \mathrm{~m}$ y $3,03 \mathrm{~m}$ para AP y $1,93 \mathrm{~m}$ y 2,04 $\mathrm{m}$ para $\mathrm{AE}$, respectivamente. En relación con el porcentaje de plantas acamadas (caídas) y quebradas (PA/PQ) las variedades BR 106 y Caiano de Sobrália y Caiano de Espírito Santo presentaron los valores más bajos con 11,12 y $12 \%$, respectivamente.

Las variedades BR 451 y Saracura presentaron los mayores valores con $26 \%$ y $24 \%$, respectivamente. 
Estos datos difieren con muchos autores, quienes han encontrado que las variedades de menor talla normalmente presentan un porcentaje menor de plantas caídas y quebradas (Paterniani 1990).

En este caso las variedades Caiano son de porte alto y no presentaron problemas con acame, debido a que el ensayo se condujo en ambiente de montañas y porque las variedades están adaptadas a dicho ambiente. En relación al peso de espigas, las variedades Eldorado y Fortaleza mostraron el mayor peso con 9.354 $\mathrm{kg} / \mathrm{ha}$ y $8.617 \mathrm{~kg} / \mathrm{ha}$, respectivamente. Las variedades que presentaran los menores valores fueran la BR 451, BR 473 y Saracura con 6.192, 6.591 y $6.654 \mathrm{~kg} / \mathrm{ha}$, respectivamente. Estos datos comprueban la eficiencia del mejoramiento participativo realizado en la propiedad del agricultor cooperante.

La variedad Eldorado, no obstante de ser desarrollada en otra región, está siendo mejorada de forma participativa desde hace más de diez años en sistema orgánico; lo mismo ocurre con Fortaleza que está siendo seleccionada hace más de diez años en la comunidad donde fueron realizados los ensayos. Cabe señalar que las variedades comerciales BR 451, BR 473 y Saracura, presentaron los valores menores, no mostraron potencial productivo en los ensayos conducidos en sistema orgánico. Probablemente ocurrió una baja adaptabilidad de esas variedades a este sistema, debido al largo proceso de selección a que las mismas fueron sometidas en sistemas convencionales. Se registraron coeficientes de variación bajos (CV\%) para todos los caracteres, lo que genera una alta confiabilidad en los datos obtenidos.

En el Cuadro 4, se presentan los datos referentes al carácter peso de las espigas $(\mathrm{kg} / \mathrm{ha})$, a partir de los ensayos realizados en los años agrícolas de 2002/2003; 2003/2004 y 2004/2005 con sus respectivas ganancias de selección y clasificación en el curso de la evaluación visual realizada por los agricultores de la comunidad de Muqui referente a la media de los tres años agrícolas. Se verificó, para el año agrícola de 2002/2003, que las variedades Caiano de Sobrália y Eldorado, sobresalieron con producciones de grano

Cuadro 4. Datos referentes al carácter peso de espigas ( $\mathrm{kg} / \mathrm{ha}$ ) de maíz, a partir de los ensayos realizados en los años agrícolas de 2002/2003, 2003/2004 y 2004/2005 con sus respectivas ganancias por selección y clasificación resultante de evaluación visual realizada por los agricultores de la comunidad de Muqui, Brasil, referente a media de los tres años agrícolas.

\begin{tabular}{|c|c|c|c|c|c|c|}
\hline Variedades & $\begin{array}{c}\text { Peso } \\
2002 / 2003\end{array}$ & $\begin{array}{c}\text { Peso } \\
2003 / 2004\end{array}$ & $\begin{array}{c}\text { Peso } \\
2004 / 2005\end{array}$ & $\begin{array}{c}\text { GS } \\
\left(\mathrm{C}_{2} / \mathrm{C}_{0}\right)^{1}\end{array}$ & $\begin{array}{c}\text { GS } \\
\left(\mathrm{C}_{2} / \mathrm{C}_{1}\right)^{2}\end{array}$ & $\begin{array}{c}\text { Clasificación visual } \\
\text { de las variedades }\end{array}$ \\
\hline Sol da Manhã & 5.700 & 7.380 & 7.880 & 2,18 & 0,50 & 4 \\
\hline Eldorado & 7.280 & 9.580 & 11.280 & 4,00 & 1,70 & 1 \\
\hline BR 106 & 6.620 & 8.320 & 8.340 & 1,72 & 0,02 & 10 \\
\hline BR 473 & 5.540 & 7.180 & 7.140 & 1,60 & $-0,04$ & 9 \\
\hline BR 451 & 5.100 & 6.100 & 7.360 & 2,26 & 1,26 & 8 \\
\hline Saracura & 5.260 & 7.680 & 7.020 & 1,76 & $-0,66$ & 7 \\
\hline Caiano de Sobrália & 7.300 & 9.060 & 9.100 & 1,80 & 0,04 & 5 \\
\hline Palha Rôxa de Santa Catarina & 5.000 & 7.980 & 10.280 & 5,28 & 2,30 & 2 \\
\hline Caiano do Espírito Santo & 5.360 & 7.360 & 8.820 & 3,46 & 1,46 & 6 \\
\hline Fortaleza & 6.680 & 8.560 & 10.580 & 3,90 & 2,02 & 3 \\
\hline Media General & 5.980 & 7.920 & 8.780 & & & \\
\hline C.V. $(\%)$ & 20,26 & 9,39 & 11,01 & & & \\
\hline DMS (5\%) & 1,041 & 0,637 & 0,829 & & & \\
\hline
\end{tabular}

${ }^{1}\left(C_{2} / C_{0}\right)$ : Media del carácter en la población mejorada y cultivada en el año agrícola de 2004/2005, denominada $C_{2}$ menos a media del carácter en el material considerado $C_{0}$ y cultivado en el año agrícola 2002/2003.

$2\left(C_{2} / C_{1}\right)$ : Media del carácter en la población mejorada y cultivada en el año agrícola de 2004/2005, denominada $C_{2}$ menos a media del carácter en el material considerado $C_{1}$ y cultivado en el año agrícola 2003/2004. 
7.280 y $7.300 \mathrm{~kg} / \mathrm{ha}$, respectivamente, mientras las variedades BR 451 y Saracura, presentaron los menores valores con 5.100 y $5.260 \mathrm{~kg} / \mathrm{ha}$. En el año agrícola de 2003/2004, nuevamente las variedades Eldorado y Caiano de Sobrália fueron las mejores con producciones de 9.580 y $9.060 \mathrm{~kg} / \mathrm{ha}$, respectivamente, mientras las variedades BR 451 y BR 473, presentaron los menores valores con 6.100 y $7.180 \mathrm{~kg} / \mathrm{ha}$.

En el año agrícola de 2004/2005, las variedades Eldorado y Fortaleza presentaron los mayores valores con 11.280 y $10.580 \mathrm{~kg} / \mathrm{ha}$, respectivamente, y las variedades Saracura y BR 473 presentaron los menores valores con 7.020 y $7.140 \mathrm{~kg} / \mathrm{ha}$, respectivamente. En estos ensayos, se pudo observar que, de una forma general, las variedades comerciales BR 451, BR 473, Saracura y BR 106 no presentaron potencial productivo en este ensayos, muy probablemente debido a la falta de adaptación de éstas al sistema orgánico de cultivo.

Las variedades que sufrieron varios ciclos de mejoramiento participativo en sistemas orgánicos como Eldorado y Fortaleza y las variedades locales Caiano de Sobrália y Palha Rôxa de Santa Catarina, se cultivan también en sistemas orgánicos, presentaron potenciales productivos altos en estos ensayos.

Observando los tres años agrícolas, se verificó una evolución tanto en la calidad del ambiente que está siendo manejado orgánicamente, con una evolución en la productividad media de los ensayos, pasando de $5.980 \mathrm{~kg} / \mathrm{ha}$ en $2002 / 2003$ a $8.780 \mathrm{~kg} / \mathrm{ha}$ en $2004 / 2005$, como también, en la conducción de los ensayos, donde tuvimos un coeficiente de variación cercano al $20 \%$ para el año agrícola de 2002/2003, con una reducción considerable para los ensayos subsecuentes, cuando el CV se redujo a un $10 \%$; esto indica una alta confiabilidad en los resultados de los ensayos.

En la tentativa de entender un poco la evolución de las variedades en relación a las ganancias por selección (GS), se pudo verificar que cuando comparamos el Ciclo 2 de las variedades que fueron cultivadas en el año agrícola de 2004/2005, se encontraron valores bastante acentuados para las variedades Eldorado y Palha Rôxa de Santa Catarina y valores bajos para las variedades comerciales BR 473 y BR 106.

Si se comparan los Ciclos 2 y 1 estas diferencias disminuyen, pero nuevamente las variedades que presentaron las mayores ganancias fueron el Eldorado, Palha Rôxa de Santa Catarina y la Fortaleza y nuevamente los menores valores los mostraron las variedades mejoradas, se destacaron BR 106 y BR 451. Nuevamente estos datos confirman la poca adaptabilidad de las variedades comerciales para este sistema de cultivo. Lo contrario ocurrió entre las variedades obtenidas con mejoramiento participativo y las variedades locales mejoradas por las comunidades. En la Figura 1 se observa la evolución del mejoramiento en las variedades Eldorado y Fortaleza en relación con las variedades comerciales BR 106 y BR 473.

El potencial de las variedades locales Caiano de Sobrália y Palha Rôxa de Santa Catarina, así como de otras variedades locales fue presentado por Machado et al. 1998a, donde se demostró el alto potencial productivo de estas variedades en diferentes regiones del Brasil. Variedades locales y variedades en proceso de mejoramiento participativo, presentan un alto potencial de adaptación local observado por la adaptación a sistemas ecológicos de producción, resistencia y o tolerancia a diferentes tipos de estrés, tales como sequías prolongadas, aluminio, nitrógeno y fósforo (Machado et al. 1998b; Machado et al. 1999; Machado 2000; Machado et al. 2001; Machado y Fernandes 2001; Machado et al. 2003; Machado y Machado 2004).

Otro dato interesante se refiere a la evaluación hecha por la Comunidad a partir de la evaluación visual de las espigas, donde las tres primeras seleccionadas fueron las variedades Eldorado, Palha Rôxa de Santa Catarina y Fortaleza y las peores fueron la BR 106, BR 451 y la BR 473. Estos resultados apoyan los resultados obtenidos a partir de las evaluaciones agronómicas de los ensayos. Esta evaluación participativa fortalece el entendimiento de la comunidad en relación con la diversidad genética de la cultura y estimula el debate sobre las diferentes formas que estas variedades pueden utilizarse. Este tipo de evaluación se viene realizando con resultados bastante satisfactorios (Machado et al. 2002).

El desarrollo de variedades vía mejoramiento participativo, desempeña un importante papel en la conquista por la soberanía alimentaria de las comunidades de pequeños agricultores y es un fuerte componente para su empoderamiento. Actualmente, en diferentes regiones del mundo, trabajos de mejoramiento participativo han tenido fuerte impacto en el desarrollo comunitario 


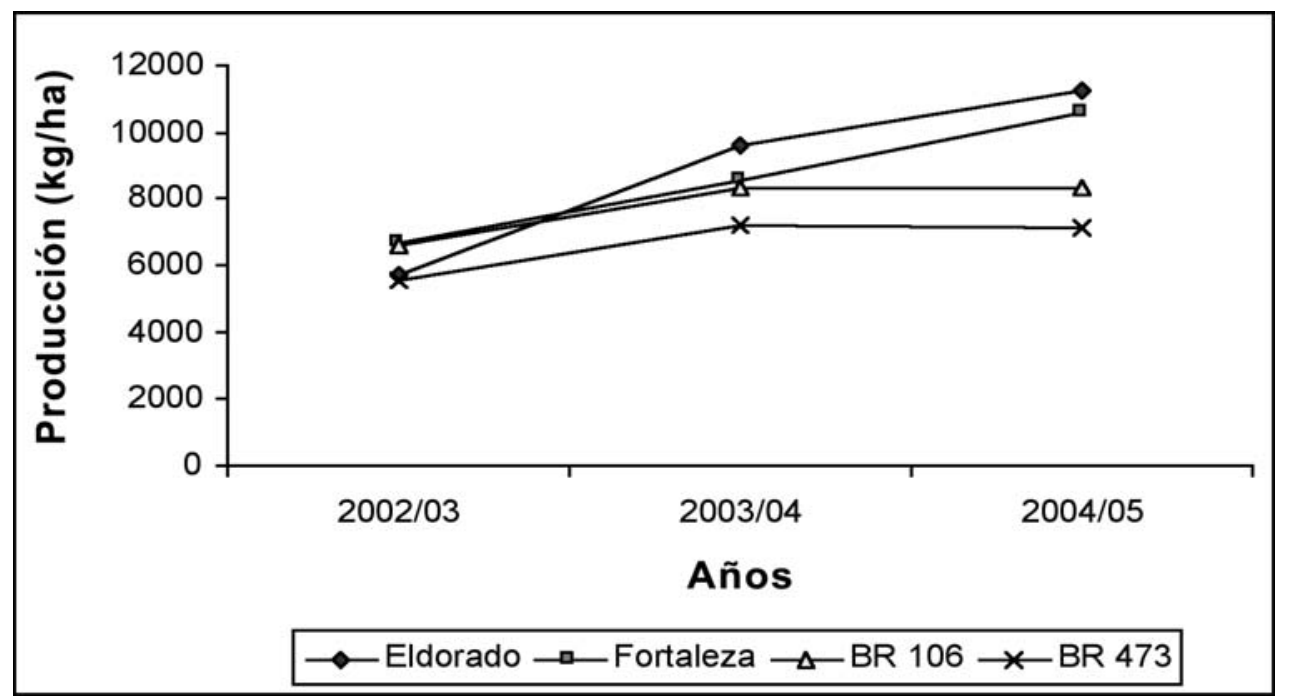

Figura 1. Producción de granos en kg/ha entre las variedades de maíz Eldorado, Fortaleza, BR 106 y BR 473, en Muqui, Brasil, evaluadas en los años agrícolas de 2002/03; 2003/04 y 2004/05.

(Machado 2000; Machado y Fernandes 2001; Almekinders y Elings 2001; Sperling et al. 2001; Witcombe et al. 2001; Smith et al. 2001; Bänziger y Cooper 2001; Machado et al. 2002; Machado y Machado 2003).
En el Cuadro 5 se presentan los datos referentes a la comercialización y a la producción de semillas comunitarias en el período de 2001 a 2005. Estos datos resaltan la evolución de la comunidad en relación al

Cuadro 5. Datos referentes a la comercialización y a la producción de semillas comunitarias de maíz en Muqui, Brasil, en el período de 2001 a 2005.

\begin{tabular}{lccccc}
\hline & \multicolumn{5}{c}{ Años agrícolas } \\
& $\mathbf{2 0 0 1}$ & $\mathbf{2 0 0 2}$ & $\mathbf{2 0 0 3}$ & $\mathbf{2 0 0 4}$ & $\mathbf{2 0 0 5}$ \\
\hline Cantidad de semillas compradas (t) (1) & 8,0 & 0,0 & 0,0 & 0,0 & 0,0 \\
Cantidad de semillas producidas (kg) & $200(2)$ & 1.700 & 5.000 & 7.400 & 10.000 \\
Agricultores participantes & 01 & 35 & 37 & 45 & 55 \\
Área cultivada (ha) & 0,5 & 1,0 & 15,0 & 22,0 & 27,5 \\
Costo relativo a la compra de semillas (R\$) & 20.000 & 0,0 & 0,0 & 0,0 & 0,0 \\
Lucro relativo a la venta de semillas (R\$) & Doação (3) & 3.400 & 10.000 & 18.500 & 30.000 \\
Variedades mejoradas (4) (cantidad) & 01 & 02 & 03 & 03 & 03 \\
Variedades producidas (5) (cantidad) & 01 & 03 & 04 & 04 & 06 \\
\hline
\end{tabular}

1 Semillas adquiridas por la municipalidad y distribuidas a los agricultores. Por ocasión de esa distribución, quedó acertado que la Municipalidad no más adquiriría semillas, pero, daría apoyo técnico y financiero para que los agricultores pasen a producir la propia semilla. De ahí surgió el Ensayo Nacional de Maíz Variedad y la producción comunitaria de semillas.

2 Semillas producidas por el agricultor Claudio Evanes B. Candido, fruto del trabajo iniciado en 1993.

3 Donación hecha por la municipalidad.

4 Las variedades que están siendo mejoradas son Sol da Manhã , Fortaleza y Palha Rôxa de Santa Catarina.

5 Las variedades de maíz que están siendo producidas son Sol da Manhã , Fortaleza, Palha Rôxa de Santa Catarina, EMCAPA 202, Caiano y Pico de Oro. 
trabajo participativo con maíz. En el año 2001, ocho toneladas de semillas se adquirieron por la comunidad con el apoyo de la municipalidad, y en esta misma época se inició el trabajo de investigación participativa, se observa que en los años subsiguientes no hubo necesidad de comprar más semillas.

En el año 2001, sólo un agricultor producía en promedio $200 \mathrm{~kg}$ semillas, con el paso del tiempo la comunidad incrementó esta producción y hoy se producen $10.000 \mathrm{~kg}$, aproximadamente. Hubo una evolución en la participación de la comunidad, pasando de un agricultor productor en 2001 a 55 agricultores productores en 2005, con un aumento del área cultivada de 0,5 ha en 2001 a 27,5 ha en 2005. La comunidad conquistó la autonomía en relación con las semillas de maíz, no habiendo más gastos en compra de semillas, además de alcanzar un beneficio a través de la venta de semillas estimado en $\mathrm{R} \$ 30.000,00$ (aproximadamente US\$14,000.00).

En la actualidad los agricultores trabajan en el mejoramiento de tres variedades en función de los resultados de los ensayos y produciendo semillas en seis variedades diferentes. El resultado de este trabajo llevó a la comunidad a promover un trabajo similar con otros cultivos como frijol y arroz. El empoderamiento de la comunidad de Muqui es una realidad y sirve de ejemplo para otras comunidades en el Brasil.

Actualmente, se desarrollan trabajos de fortalecimiento comunitario, a partir del manejo de la diversidad genética en maíz, con base en la estrategia del mejoramiento participativo, en diferentes regiones de Brasil como en Seropédica (RJ) (Machado y Fernández 2001), en el oeste del estado de Santa Catarina (Canci 2002; Canci et al. 2004) y en el sudoeste de Paraná (Tardin et al. 2005), además de otras iniciativas en curso en el norte del estado de Minas Gerais y en la región centro-oeste del Brasil, en el estado de Goiás y en el Distrito Federal, todavía no sistematizados y sintetizados.

\section{CONCLUSIONES}

En sistemas orgánicos, se obtuvieron variedades locales con alto potencial de producción como: Caiano de Sobrália y Palha Rôxa de Santa Catarina.
El mejoramiento participativo es eficiente y ayudó a incrementar la productividad y en la adaptación de las variedades Eldorado y Fortaleza, en ambiente con manejo orgánico.

El desarrollo de actividades de mejoramiento participativo permitió la conquista de la autonomía por la comunidad en lo que se refiere a la producción de semillas de maíz (Zea mays L.), estableciendo las bases para su empoderamiento.

\section{AGRADECIMIENTOS}

Los autores agradecen a los agricultores de la comunidad Fortaleza y la Municipalidad de Muqui, que mucho contribuyeron para el éxito de la conducción de este trabajo.

\section{LITERATURA CITADA}

ALMEKINDERS, C.J.M.; ELINGS, A. 2001. Collaboration of farmers and breeders: participatory crop improvement in perspective. Euphytica, Wageningen, 122: 425-438.

BANZIGER, M.; COOPER, M. 2001. Breeding for low input conditions and consequences for participatory plant breeding: Examples from tropical maize and wheat. Euphytica, Wageningen 122: 503-519.

CANCI, A. 2002. Sementes crioulas: construindo soberania A semente na mão do Agricultor. São Miguel do Oeste: Mclee. 161p.

CANCI, A.; VOGT, G.A.; CANCI, I.J. 2004. A diversidade das espécies crioulas em Anchieta - SC: diagnóstico, resultados de pesquisa e outros apontamentos para a conservação da agrobiodiversidade. São Miguel do Oeste: Mclee. 112p.

CONSULTIVE GROUP ON INTERNATIONAL AGRICULTURAL RESEARCH. 1999a. Crossing perspective: farmers and scientists in participatory plant breeding. Program on participatory research and gender analysis (PRGA) - Consultive Group on International Agricultural Research (CGIAR). Cali, Colombia. 49 p.

AGRONOMÍA MESOAMERICANA 17(3): 393-405. 2006 
CONSULTIVE GROUP ON INTERNATIONAL AGRICULTURAL RESEARCH. 1999b. Systemwide program on participatory research and gender analysis for technology development and Institutional Innovation (PRGA Program). Annual Report. Cali, Colombia.

GOMES, F.P. 1982. Estatística Experimental. 10a edição, ESALQ/USP. Piracicaba, SP. 430 p.

MACHADO, A.T.; MAGALHÃES, J.R.; MAGNAVACA, R.; SILVA, M.R. 1992. Determinação das atividades de enzimas envolvidas no metabolismo do nitrogênio em diferentes genótipos de milho. Revista Brasileira de Fisiologia Vegetal 4(1): 45-47.

MACHADO, A.T. 1998. Resgate e caracterização de variedades locais de milho. In: Soares, A.C.; Machado, A.T.; Silva, B.M.; Weid, von der J.M. eds. Milho Crioulo: conservação e uso da biodiversidade. Rio de Janeiro: AS-PTA. p. 82-92.

MACHADO, A.T.; PEREIRA, M.B.; PEREIRA, M.E.; MACHADO, C.T. de T.; MÉDICE, L.O. 1998a. Avaliação de variedades locais e melhoradas de milho em diferentes regiões do Brasil. In: Soares, A.C.; Machado, A.T.; Silva, B.M.; Weid, von der J.M. eds. Milho Crioulo: conservação e uso da biodiversidade. Rio de Janeiro: AS-PTA. p. 93-106.

MACHADO, A.T.; MACHADO, C.T. de T.; FURLANI, P.R. 1998b. Avaliação e caracterização de variedades locais de milho para condições adversas de ambiente. In: Soares, A.C.; Machado, A.T.; Silva, B.M.; Weid, von der J.M. eds. Milho Crioulo: conservação e uso da biodiversidade. Rio de Janeiro: AS-PTA, p. 151-178.

MACHADO, A.T. 2000. The collaborative development of stress-tolerant maize varieties in Rio de Janeiro, Brazil. In: Almekinders, C; de Boef, W. eds. Encouragin Diversity: the conservation and development of plant genetic resources. London: Intermediate Technology Publications. p. 199-202.

MACHADO, A. T.; FERNANDEZ, M. 2001. Participatory maize breeding for low nitrogen tolerance. Euphytica, Wageningen 122: 567-573.

MACHADO, A.T.; MACHADO, C.T.T.;COELHO, C.H.M. 2002. Manejo da diversidade genética do milho e melhoramento participativo em comunidades agrícolas nos estados do Rio de Janeiro e Espírito Santo. Planaltina: Embrapa Cerrados. 22p. (Embrapa Cerrados. Boletim de Pesquisa e Desenvolvimento, 32).

MACHADO,A.T.; MACHADO,C.T.T.; MIRANDA,G.V.; COELHO,C.H.M.; GUIMARÃES, L.J.M. 2003. Resposta de variedades de milho a níveis e fontes de nitrogênio. Planaltina: Embrapa Cerrados. 27p. (Embrapa Cerrados. Boletim de Pesquisa e Desenvolvimento, 93).

MACHADO, A.T.; MACHADO, C.T.T. 2003. Melhoramento vegetal participativo com ênfase na eficiência nutricional. Planaltina: Embrapa Cerrados. 39p. (Documentos/Embrapa Cerrados, 104).

MACHADO, A.T.; MACHADO, C.T.T. 2004. Management of genetic diversity of maize in agricultural communities in Brazil. In: Badejo, M.A.; Togun, A.O. eds. College Press \& Publishers Ltd. Strategies and Tactics of Sustainable Agriculture in the Tropics 2: 181-195.

MACHADO, C.T.T.; GUERRA, J.F.M.; ALMEIDA, D.L.; MACHADO, A.T. 1999. Variabilidade entre genótipos de milho para eficiência no uso de fósforo. Bragantia, Campinas 58(1): 109-124.

MACHADO, C.T. de T.; FURLANI, A.M.C.; MACHADO, A.T. 2001. Índices de eficiência de variedades locais e melhoradas de milho ao fósforo. Bragantia, Campinas, 60(30): 225-238.

NASPOLINI FILHO, V.; GOMES, E.E.; VIRNA, R.T.; MÔRO, J.R. 1981. General and specific combining ability for field in a diallel cross among 18 maize populations. Revista Brasileira de Genética, Ribeirão Preto 4(4): 571-577.

PATERNIANI, E. 1990. Maize breeding in the tropics. Critical Reviews in Plant Sciences, Oxford, 9: 125-154.

SMITH, M. E.; CASTILLO, F.; GÓMEZ, F. 2001. Participatory plant breeding with maize in Mexico and Honduras. Euphytica, Wageningen 122: 551-565.

SOARES, A.C.; MACHADO, A.T.; SILVA, B.M.; VON DER WEID, J.M. 1998. Milho crioulo: conservação e uso da biodiversidade. Rio de Janeiro: AS PTA. 185 p. 
SPERLING, L.; ASHBY, J.A.; SMITH, M.E.; WELTZIEN, E.; McGUIRE, S. 2001. A framework for analyzing participatory plant breeding approaches and results. Euphytica, Wageningen 122: 439-450.

TARDIN, J.M.; JANTARA, A.E.; MOREIRA, R.M.P.; FERREIRA, J.M. 2005. Organização social dos agricultores da região Centro Sul do Paraná em busca da autonomia, sustentabilidade e desenvolvimento da agricultura familiar. In: Mota, D.M.; Schmitz, H.;Vasconcelos, H.E.M. eds. Agricultura Familiar e Abordagem Sistêmica. Aracaju, SE: Sociedade Brasileira de Sistemas de Produção. 398 p.
VENCOVSKY, R. 1987. Herança quantitativa. In: Paterniani, E.; Viégas, G.P. eds. Melhoramento e Produção de milho. Vol. 1. Fundação Cargill, Campinas, SP. p.137-214.

VERNOOY, R.; SONG, Y. 2004. New approaches to supporting the agricultural biodiversity important for sustainable rural livelihoods. International Journal of Agricultural Sustainability 2(1): p.55-66.

WITCOMBE, J.R.; JOSHI, K.D.; RANA, R.B.; VIRK, D.S. 2001. Increasing genetic diversity by participatory varietal selection in high potential production systems in Nepal and India. Euphytica, Wageningen 122: 575-588. 